\title{
A Hipótese do Pólo Comum entre a Pré-Eclâmpsia e o Diabetes Gestacional
}

$\mathrm{O}^{\prime \prime}$ DiABETES MELITO GESTACIONAL (DMG) e a pré-eclâmpsia são condições clínicas da gravidez que estão associadas a maior risco materno-fetal (1).

Na gestante, o DMG aumenta o risco de prematuridade, parto cesariana, de evolução para diabetes tipo 2 , enquanto no feto ocorre maior risco de macrossomia, hiperinsulinemia e obesidade ao longo da vida $(2,3)$. Na préeclâmpsia, assim como no DMG, também ocorre aumento do risco de prematuridade e de parto cesáreo, além do comprometimento renal e da possível evolução para hipertensão arterial crônica (4). O recém-nascido da gestante com pré-eclâmpsia está mais propenso a apresentar a síndrome do desconforto respiratório e o retardo de crescimento intra-uterino (5).

O DMG incide de forma global em, aproximadamente, $5 \%$ a $10 \%$ das gestações (6) e, em nosso meio, de acordo com os critérios da Organização Mundial da Saúde (OMS), corresponde a 7,6\% das gestações (7). Sua incidência varia consoante os diferentes grupos étnicos e raciais, porquanto as mulheres asiáticas e de origem hispânica são as que possuem as maiores taxas de DMG. A pré-eclâmpsia é ainda a principal causa de morte materna (8). No Brasil, representa $37 \%$ dos óbitos maternos (3), e a sua prevalência é de $6 \%$ a $9 \%$ das gestações, dependendo da etnia. Dada a importância dessas duas patologias que, muitas vezes, são encontradas em associação e complicam a evolução da gestação, o conhecimento dos fatores de risco que estão presentes é de fundamental importância para o entendimento do mecanismo etiológico e para o planejamento de medidas de prevenção.

Um dos artigos dessa edição dos ABE\&M: Diabetes gestacional e préeclâmpsia: antecedentes comuns?, comparou os fatores de risco para diabetes melito gestacional e pré-eclâmpsia na população brasileira e demonstrou que no Brasil (9) o DMG e a pré-eclâmpsia compartilham os mesmos fatores de risco. O trabalho de Wendland e cols. revelou que a freqüência dessas patologias aumenta com a idade e a adiposidade e diminui com o grau de paridade. Na população estudada, foram encontradas $3,1 \%$ de pré-eclâmpsia e eclâmpsia, embora pouco diferente dos relatos da literatura ( $5 \%$ a $10 \%)$, os autores mencionam a possibilidade do não-relato do diagnóstico em alguns centros. Foram diagnosticados 7,5\% de DMG por meio do TOTG de 2 horas com 75 g no grupo de gestantes estudadas, dados estes semelhantes aos da literatura.

A possibilidade de um elo comum entre essas patologias tem sido discutida na literatura (5). A resistência insulínica - condição conhecida pelo seu potencial de causar disfunção endotelial e ativação inflamatória crônica, com aumento do risco cardiovascular e de evolução para o diabetes tipo 2 - parece ser esse elo. Portanto, o trabalho, ao trazer dados específicos da nossa população, traz subsídios valiosos para o planejamento de estudos que elucidem os mecanismos que determinam a evolução para uma ou outra destas patologias gestacionais.

\section{editorial}

DeNISE ReIS Franco

Médica Endocrinologista Pesquisadora do Centro de Pesquisa Clínica (CPclin), Coordenadora de Educação em Diabetes da Associação de Diabetes Juvenil (ADJ), São

Paulo, SP, Brasil. 
Outro aspecto ainda controverso é a relação do hábito de fumar e sua associação com a incidência de DMG e pré-eclampsia (9). Esse tema também é discutido no trabalho de Wendland e cols. Na literatura são demonstrados diferentes resultados, dependendo do tempo de exposição à nicotina. A exposição aguda teria efeito diabetogênico pelo aumento da resistência insulínica mediado pela liberação de catecolaminas por meio da estimulação do sistema nervoso simpático (10). Entretanto, o uso transitório da nicotina na gravidez parece não exercer efeito na secreção de insulina e, a longo prazo, permanece duvidosa sua ação no metabolismo de glicose. Mesmo que este dado não fosse controverso, certamente não seria recomendável fumar como caráter preventivo.

Os mecanismos para desenvolvimento da doença cardiovascular e o risco associado ao diabetes gestacional ainda precisam ser estabelecidos. O seguimento destas mulheres que apresentaram diabetes gestacional e das que desenvolveram pré-eclâmpsia poderá no futuro esclarecer a inter-relações entre estas duas patologias da gravidez.

\section{REFERÊNCIAS}

1. Xiong X, Saunders LD, Wang FL, Demianczuk NN. Gestational diabetes mellitus: prevalence, risk factors, maternal and infant outcomes. Int J Gynaecol Obstet. 2001;75(3):221-8.

2. Montenegro Jr. RM, Paccola GMFG, Faria CM, et al. Evolução materno-fetal de gestantes diabéticas seguidas no HC-FMRPUSP no período de 1992-1999. Arq Bras Endocrinol Metab. 2001;45(5):467-74.
3. Laurenti R, Jorge MHP, Gotlieb SLD. Maternal mortality in Brazilian state capitals: some characteristics and estimates for an adjustment factor. Rev Bras Epidemiologia. 2006;7(4):449-60.

4. Report of the National High Blood Pressure Education Program Working Group on High Blood Pressure in Pregnancy. Am J Obstet Gynecol. 2000;183(1):S1-22.

5. Villar J, Carroli G, Wojdyla D, Abalos E, Giordano D, Ba'aqeel $\mathrm{H}$, et al. Preeclampsia, gestational hypertension and intrauterine growth restriction, related or independent conditions? Am J Obstet Gynecol. 2006;194(4):921-31.

6. Metzger BE, Buchanan TA, Coustan DR, Leiva A, Dunger DB, Hadden DR, et al. Summary and recommendations of the Fifth International Workshop-Conference on Gestational Diabetes Mellitus. Diabetes Care. 2007; 30(2):S251-60.

7. Schmidt MI, Matos MC, Reichelt AJ, Forti AC, de Lima L, Duncan BB. Prevalence of gestational diabetes mellitus - do the new WHO criteria make a difference? Brazilian Gestational Diabetes Study Group. Diabet Med. 2000;17(5):376-80.

8. ACOG Committee on Practice Bulletins Obstetrics: ACOG practice bulletin. Diagnosis and management of preeclampsia and eclampsia. Obstet Gynecol. 2002; 99(1):159-67.

9. Wendland EMDR, Duncan BB, Belizán JM, Vigo A, Schmidt MI. Gestational diabetes and pre-eclampsia: common antecedents? Arq Bras Endocrinol Metab. 2008;52-975-84.

10. Terry PD, Weiderpass E, Östenson CG, Cnattingius S. smoking and the risk of gestational and pregestational diabetes in two consecutive pregnancies. Diabetes Care. 2003;26:2994-8.

Endereço para correspondência:

Denise Reis Franco

Centro de Pesquisa Clínica (CPCLIN)

Rua Goiás, 193

01233-020 São Paulo, SP

e-mail: d9franco@terra.com.br 